punto org

Collana diretta da Luigi Maria Sicca

39 


\author{
GiampaOlo Viglia \\ ANNA ChIARA INVERNIZZI
}

\title{
IL RUOLO DELL'HUBRIS \\ NELLA GESTIONE \\ IMPRENDITORIALE
}

\author{
Prefazione \\ Chiara Mauri
}

Editoriale Scientifica

Napoli 
Tutti i diritti sono riservati

(C) 2018 Editoriale Scientifica s.r.l.

Via San Biagio dei Librai, 39

80138 Napoli

www.editorialescientifica.com

info@editorialescientifica.com

ISBN 978-88-9391-278-5 


\section{Indice}

9 Prefazione. La necessità di supporto nelle decisioni Chiara Mauri

13 Introduzione

19 1. L'HUBRIS MANAGERIALE

19 1.1. Errori di valutazione cognitivi

23 1.2. L'approccio psicologico

24 1.3. La gestione delle imprese

32 1.4. L'inizio dell'eccesso di sicurezza: le scelte di investimento

33 1.5. L'hubris nell'area gestionale

35 1.6. Gli effetti delle distorsioni comportamentali sulle decisioni operative

36 1.7. L'hubris nelle piccole e medie imprese

41 2. L'HUBRIS MANAGERIALE DEGLI IMPRENDITORI: EVIDENZE EMPIRICHE

41 2.1. Introduzione

43 2.2. Rassegna bibliografica

43 2.2.1. Eccesso di sicurezza

45 2.2.2. Quali fattori potrebbero portare a una situazione di fallimento aziendale nelle PMI

49 2.2.3. Riconoscere l'attitudine di eccesso di sicurezza dell'imprenditore

51 2.3. Ipotesi

52 2.4. Metodo di ricerca: Analisi empirica per testare l'eccesso di sicurezza 
52

53

54

54

56

60

67

67

68

68

69

72

75

75

77

80

85

88

89

91

93

93

97

99

2.4.1. Dati

2.4.2. Campioni

2.5. Analisi e risultati

2.5.1. Le tre principali variabili dipendenti

2.5.2. L'incidenza delle variabili indipendenti 2.6. Discussione e Conclusioni

3. INDAGINE QUALITATIVA E COMMENTI CONCLUSIVI SU HUBRIS NEL BUSINESS

3.1. Razionale

3.2. Indagine qualitativa

3.3. Collezione dei dati

3.4. Risultati

3.5. Implicazioni

4. L'IMPATTO DEL LIVELLO DI SICUREZZA DECISIONALE SULLA PERFORMANCE

4.1. Introduzione

4.2. Framework teorico e Ipotesi

4.3. Studio 1

4.4. Studio 2

4.5. Discussione

4.6. Conclusioni

4.7. Riflessioni

5. LE IMPLICAZIONI PER LE PICCOLE E MEDIE IMPRESE

5.1. L'impatto dei bias decisionali nelle decisioni di investimento

5.2. L'impatto dei fenomeni qualitativi nelle decisioni di finanziamento

5.3. Implicazioni

Conclusione 
111 Ringraziamenti

113 Bibliografia

129 Elenco delle Immagini

131 Elenco delle Tabelle

133 Hanno scritto nella Collana punto org 


\section{Prefazione \\ La necessità di supporto nelle decisioni}

Chiara Mauri*

Hubris, che deriva dal greco hybris, è un topos ricorrente nella mitologia e nella letteratura greca, dove significa tracotanza, arroganza, alterigia, supponenza e presunzione. Tutte queste espressioni hanno in comune un comportamento eccessivo, negativo perché è attuato non tanto per la sua utilità quanto per il piacere che suscita in chi lo mette in atto.

L'hubris è un comportamento tipico degli imprenditori? Produce risultati positivi o negativi? Le risposte in letteratura sono controverse, nonostante taluni abbiamo tacciato l'hubris come un comportamento socialmente irresponsabile e persino catastrofico. Se da un lato una certa presunzione spinge l'imprenditore a porsi traguardi di crescita sfidanti magari superando ostacoli impegnativi, dall'altro la sopravvalutazione delle proprie capacità può condurre a situazioni di frustrazione e rinuncia quando $i$ risultati sono inferiori alle aspettative. L'hubris è infatti misurato come la differenza tra aspettative e risultati raggiunti.

Giampaolo Viglia e Anna Invernizzi cercano di fare chiarezza sull'impatto dell'hubris e lo indagano in due ambiti, separati ma con molti elementi in comune: le piccole e medie imprese (PMI) italiane e le donne. Leggendo il libro, e in particolare i capitoli dedicati agli imprenditori, si matura una riflessione sul caratte-

* Professore ordinario di Economia e Gestione delle Imprese, Università della Valle d'Aosta. 
re difficile di tanti imprenditori: chiunque viva la quotidianità del lavoro con un imprenditore, di successo e non, non può non avere sperimentato in prima persona l'hubris, che si manifesta in tanti comportamenti quotidiani che hanno in comune una parola: "Io", soprattutto in situazioni di performance superiori alle aspettative. In un ambito diverso, ma con molte analogie, le donne che praticano uno sport tendono invece a sottovalutare le loro performance perché temono di più il fallimento; dal mondo dello sport a quello delle imprese dirette da donne il passo è rapido: le imprese dirette da donne fanno previsioni più realistiche.

Il tema dell'hubris è poco discusso nella letteratura italiana sulle PMI, nonostante sia così pregnante nella cultura imprenditoriale: per questo vale la pena leggere la presente monografia. Le pagine sono ricche di spunti interessanti, che suscitano riflessioni e desiderio di approfondire. Per esempio, assumere un manager molto sicuro di sé può portare gli altri collaboratori a lavorare di più: se questo è vero, si pensi all'impatto che ciò può avere sul sistema incentivante e sulla sua equità. Che cosa deve premiare un incentivo? I risultati raggiunti o la capacità di far lavorare gli altri? E allora come deve essere ripartito tra i "giocatori"?

L'indagine empirica svolta su un campione di imprenditori rivela risultati sorprendenti per chi non è esperto di hubris. L'hubris è stato misurato come differenza percentuale tra quanto previsto e quanto ottenuto relativamente a tre indicatori: EBITDA, capitale netto e costi dei prestiti. L'indagine è stata svolta su un campione di più di 250 piccole imprese italiane. Ebbene, gli imprenditori: a) sovrastimano l'EBITDA di circa l'11\%, b) sovrastimano il capitale proprio di circa il $10 \%$, e c) sottostimano il costo del debito dell'11\%. Ci sono fattori che correggono positivamente l'hubris, come l'età degli imprenditori, la presenza di donne, il livello di istruzione degli stessi imprenditori, l'adozione di un sistema informativo integrato. Insomma, man mano si scende nei dettagli dell'analisi si colgono tanti dettagli che chiunque abbia un poco di famigliarità con il mondo delle pic- 
cole imprese non può non avere vissuto in prima persona senza farvi troppo caso.

Per fare ulteriore luce sul fenomeno dell'hubris, un piccolo numero dei medesimi imprenditori è stato poi invitato a partecipare a due focus group, durante i quali gli imprenditori dovevano scrivere i loro pensieri e comporre un collage che rappresentasse l'assunzione delle decisioni strategiche nelle loro imprese. Le due espressioni più ricorrenti sono "soli" e "successo": "Sono solo quando devo decidere ... e ho un grandissimo fiuto per le scelte".

Risultati altrettanto interessanti sono quelli relativi alle differenze di genere e a comportamenti opposti a quelli dell'hubris, alla relazione tra fiducia e prestazione sportiva, e anche alla relazione tra prestazione e felicità. In questo caso sono state osservate 60 giocatrici di calcio semi-professionali a Barcellona, alle quali è stato chiesto di dichiarare quanti goal pensavano di segnare su 10 calci di punizione e in che posizione pensavano di essere nella classifica generale della prestazione.

In conclusione, il lavoro monografico discute alcuni suggerimenti che vale la pena anticipare in questa prefazione. L'eccesso di fiducia può essere vantaggioso all'inizio di un'attività imprenditoriale, perché fornisce l'energia necessaria a superare le difficoltà. Quando l'azienda cresce e si consolida, l'hubris può tuttavia influenzare negativamente le prestazioni, perché gli imprenditori fissano obiettivi esuberanti rispetto alle risorse che hanno a disposizione; imprenditori e collaboratori più istruiti e sistemi informativi sofisticati possono correggere gli effetti negativi dell'hubris. Le donne imprenditrici sono meno tracotanti, soprattutto in contesti a prevalente vocazione maschile.

Il libro copre aree di rispettiva esperienza dei due autori, economia e gestione delle imprese per il primo autore e economia aziendale per il secondo autore. I due autori, integrando distintamente i saperi di queste due aree, riescono a suscitare l'interesse del lettore fornendo una visione olistica del fenomeno che riesce a suscitare tantissime riflessioni. 